\section{Independent and Complementary Utility of Ambulatory Electrocardiogram-Based T- Wave Alternans and Heart Rate Turbulence for Predicting Major Cardiac Events in Patients After Myocardial Infarction}

\section{To the Editor:}

We read with keen interest the excellent article by Hoshida and colleagues, ${ }^{1}$ who examined the predictive capacity of simultaneous assessment of Modified Moving Average-based T-wave alternans (MMA-TWA) and heart rate turbulence (HRT) in patients during a stable phase following myocardial infarction (MI) using 24-h ambulatory electrocardiogram (ECG) recordings. Their investigation examined prospectively the clinical utility of the independent and combined use of these markers for stratification of risk for serious cardiac events in this important cohort of patients with relatively preserved ejection fraction. As Huikuri and colleagues have emphasized, ${ }^{2}$ a significant proportion of cardiac events, including sudden cardiac death (SCD), occurs in such post-MI patients.

The methodology of the present study is based on a compelling rationale, as it incorporates assessment of 2 main factors that predispose to cardiac mortality, specifically, evidence of autonomic dysfunction by means of abnormal HRT and the presence of a vulnerable myocardial substrate in terms of TWA. HRT refers to fluctuations of sinus-rhythm cycle length after a single ventricular premature beat. It has been hypothesized that the initial heart rate acceleration is triggered by transient vagal inhibition in response to the missed baroreflex afferent input due to hemodynamically inefficient ventricular contraction, and a sympathetically mediated overshoot of arterial pressure is responsible for the subsequent heart rate deceleration through vagal recruitment. ${ }^{3,4}$ TWA provides a measure of substrate vulnerability inasmuch as it reflects temporo-spatial heterogeneity of repolarization, a property that has been closely linked to cardiac electrical instability and propensity for lifethreatening arrhythmias. ${ }^{5}$

Hoshida and colleagues reported that both MMA-TWA and HRT were independent predictors of serious cardiac events when monitored at $>2$ weeks after MI and exhibited sufficient negative predictive value to support their use in population screening. ${ }^{1}$ Additionally, the 2 markers revealed different clinical features and provided insights with respect to the modality of the cardiac events. In particular, MMA-TWA, an index of repolarization abnormality, was strongly associated with fatal arrhythmic events, including SCD, whereas HRT was highly related to death from cardiac failure. The combination of MMATWA with HRT improved prediction of cardiac mortality by $100 \%$ (from 5.7, 95\% confidence interval [CI] 2.1-15.9, $\mathrm{P}<0.0008$, to 11.4 , 95\% CI 4.6-28.6, $\mathrm{P}<0.0001$ ), and adding HRT to MMA-TWA results improved prediction of SCD, ventricular fibrillation, and sustained ventricular tachycardia by $140 \%$ (from 5.8, 95\% CI 1.6-20.8, P<0.0072 to $13.9,95 \%$ CI 3.0-66.7, $\mathrm{P}<0.0009)$. Hoshida and colleagues ${ }^{1}$ found greater improvement in risk stratification by combining these parameters than did the only 2 prior groups of investigators who have used this combination, ${ }^{6,7}$ but who did not use 24 -h ECG monitoring and enrolled fewer patients, respectively.
The present investigation also offers a number of conceptual and practical advantages of ambulatory ECG-based risk assessment using TWA and HRT. Specifically, the authors demonstrated that valuable data on cardiac risk can be obtained without an exercise protocol in patients who have physical limitations or in whom it is difficult to increase heart rate because of medications. The inability to raise heart rate as a result of $\beta$-blockade therapy accounts for half of the indeterminate test results in studies using the Spectral Method of TWA analysis. In a meta-analysis, Chan et al determined that the predictive capacity of TWA for ventricular arrhythmic events was significantly weaker in TWA studies in which $\beta$-blockade therapy was withheld than in studies performed on current medications. ${ }^{8}$ Washout of $\beta$-blockade therapy at the time of TWA testing using the Spectral Method has been proposed as a major factor accounting for the negative outcomes of the Microvolt T-wave Alternans Testing for Risk Stratification of Post-Myocardial Infarction Patients (MASTER) trial and the Sudden Cardiac Death in Heart Failure Trial (SCD-HeFT) TWA substudy. ${ }^{5}$ Thus, the capacity to perform MMA-TWA testing without withdrawal of medications, as in the present investigation, constitutes a major advantage.

We would like to raise 2 questions for the authors' consideration. First, we would like to learn more specifics about the time of ECG monitoring following MI. They mentioned that the tests were performed $>2$ weeks after the event. It would be valuable to know the precise time period in terms of mean and range of weeks when the HRT and TWA measurements were made. This is a contemporary question of considerable debate. Exner and coworkers ${ }^{6}$ found no predictivity by any of the noninvasive risk stratifiers in current use, including HRT and TWA, when assessed at 2 to 4 weeks after MI. Only when the measurements were made at 10-14 weeks was prediction found. Alternatively, do the authors believe that it would be valuable to obtain these measurements both early after MI, to identify individuals who are at risk during the recovery phase, and later, after cardiac and neural remodeling occurs, when the substrate is more stable and longterm risk can be assessed?

The second question relates to the status of the utility of HRT and TWA in guiding medical and device therapy. What information have the authors gathered to date with respect to the usefulness of these markers as therapeutic targets? Do they believe that these measurements can potentially guide rehabilitation during post-MI recovery?

We commend the authors for this landmark investigation and look forward to learning about their experience and perspective regarding the issues we have raised.

\section{Disclosures}

Dr Verrier holds a patent on T-wave alternans analysis by the Modified Moving Average method, which is licensed to GE Healthcare, Inc, and Medtronic, Inc.

Dr Schmidt holds a patent on Heart Rate Turbulence, which is licensed to GE Healthcare, Inc, and Medtronic, Inc.

\section{References}

1. Hoshida K, Miwa Y, Miyakoshi M, Tsukada T, Yusu S, Yoshino H, et al. Simultaneous assessment of T-wave alternans and heart rate turbulence on Holter electrocardiograms as predictors for serious cardiac events in patients after myocardial infarction. Circ $J$ 2013; 77: $432-438$.

2. Huikuri HV, Castellanos A, Myerburg RJ. Sudden death due to cardiac arrhythmias. N Engl J Med 2001; 345: 1473-1482.

3. Lin LY, Lai LP, Lin JL, Du CC, Shau WY, Chan HL, et al. Tight mechanism correlation between heart rate turbulence and baroreflex 
sensitivity: Sequential autonomic blockade analysis. J Cardiovasc Electrophysiol 2002; 13: 427-431.

4. Bauer A, Malik M, Schmidt G, Barthel P, Bonnemeier H, Cygankiewicz I, et al. Heart rate turbulence: Standards of measurement, physiological interpretation, and clinical use: International Society for Holter and Noninvasive Electrocardiology Consensus. $J \mathrm{Am}$ Coll Cardiol 2008; 52: 1353-1365.

5. Verrier RL, Klingenheben T, Malik M, Exner D, Hohnloser S, Ikeda T, et al. Microvolt T-wave alternans: Physiologic basis, methods of measurement, and clinical utility: Consensus guideline by the International Society for Holter and Noninvasive Electrocardiology. $J \mathrm{Am}$ Coll Cardiol 2011; 44: 1309-1324.

6. Exner DV, Kavanagh KM, Slawnych MP, Mitchell LB, Ramadan D, Aggarwal SG, et al. Noninvasive risk assessment early after a myocardial infarction: The REFINE study. J Am Coll Cardiol 2007; 50: $2275-2284$.

7. Ren LN, Fang XH, Ren LD, Gong J, Wang YQ, Qi GX. Ambulatory ECG-based T-wave alternans and heart rate turbulence can predict cardiac mortality in patients with myocardial infarction with or without diabetes mellitus. Cardiovasc Diabetol 2012; 11: 104111.

8. Chan PS, Gold MR, Nallamothu BK. Do beta-blockers impact microvolt T-wave alternans testing in patients at risk for ventricular arrhythmias? A meta-analysis. J Cardiovasc Electrophysiol 2010; 21: $1009-1014$.

Richard L. Verrier, PhD

Beth Israel Deaconess Medical Center, Harvard Medical School, Boston, MA, USA

Georg Schmidt, MD

Erste Medizinische Klinik, Klinikum rechts der Isar, Technische Universität München, Munich, Germany

(Released online January 9, 2013) 